\title{
Dynamics of the flood response to slow-fast landscape-climate feedbacks
}

\author{
R. A. P. Perdigão and G. Blöschl \\ Institute of Hydraulic Engineering, Vienna University of Technology, Vienna, Austria \\ Correspondence to: R. A. P. Perdigão (perdigao@hydro.tuwien.ac.at)
}

Received: 11 March 2015 - Accepted: 11 March 2015 - Published: 11 June 2015

\begin{abstract}
The dynamical evolution of the flood response to landscape-climate feedbacks is evaluated in a joint nonlinear statistical-dynamical approach. For that purpose, a spatiotemporal sensitivity analysis is conducted on hydrological data from 1976-2008 over 804 catchments throughout Austria, and a general, data-independent nonlinear dynamical model is built linking floods with climate (via precipitation), landscape (via elevation) and their feedbacks. These involve nonlinear scale interactions, with landform evolution processes taking place at the millennial scale (slow dynamics), and climate adjusting in years to decades (fast dynamics). The results show that floods are more responsive to spatial (regional) than to temporal (decadal) variability. Catchments from dry lowlands and high wetlands exhibit similarity between the spatial and temporal sensitivities (spatiotemporal symmetry) and low landscape-climate codependence, suggesting they are not coevolving significantly. However, intermediate regions show differences between those sensitivities (symmetry breaks) and higher landscapeclimate codependence, suggesting undergoing coevolution. The break of symmetry is an emergent behaviour from nonlinear feedbacks within the system. A new coevolution index is introduced relating spatiotemporal symmetry with relative characteristic celerities, which need to be taken into account in hydrological space-time trading. Coevolution is expressed here by the interplay between slow and fast dynamics, represented respectively by spatial and temporal characteristics. The dynamical model captures emerging features of the flood dynamics and nonlinear landscape-climate feedbacks, supporting the nonlinear statistical assessment of spatiotemporally asymmetric flood change. Moreover, it enables the dynamical estimation of flood changes in space and time from the given knowledge at different spatiotemporal conditions. This study ultimately brings to light emerging signatures of change in floods arising from nonlinear slow-fast feedbacks in the landscape-climate dynamics, and contributes towards a better understanding of spatiotemporal flood changes and underlying nonlinearly interacting drivers.
\end{abstract}

\section{Introduction}

Floods are known to respond to a diversity of controls of natural and anthropogenic nature (Hall et al., 2014). Within the natural setting alone, several processes influence the flood behaviour in different manners. One approach to taking these into account is to evaluate the flood sensitivity to each process and consider the overall response as the superposition of these individual controls. However, the dynamical interference between drivers is also a control on its own and is not captured by traditional evaluation methods. Fortunately though, novel methodologies begin to emerge quantifying nonlinear synergies between dynamical processes, such as the framework devised and implemented by Pires and Perdigão (2015) for the broader geosciences, introducing measures of non-linear interaction information as quantifiers of dynamic and statistical interference or synergy between processes, along with a physical ground to the emerging structures that ensue.

Another issue with floods and the overall hydrological system in general lies in the non-equilibrium dynamics at play, resulting in unstable dynamical behaviour and corresponding non-stationary statistical footprint. In other words, analysis and modelling methods assuming a stationary hydrological system, while workable in shorter time scales, fail to cap- 
ture regime transitions over the long run. One might then just capture the overall long-term statistics, but then the faster dynamics taking place at shorter time scales are missed out. Even if the interest is only on the longer time scales, the dynamics taking place at that level are also affected by shorterterm dynamics, due to scale interactions. It is thus of interest to take into account the slow-fast dynamics taking place in the hydrological system, be our interest on either short, long or intermediate scale dynamics. The fundamental idea is that the dynamics at one scale is a function of the dynamics at other scales as well.

Due to the general lack of long-term records, long-term dynamics are often inferred via the spatial variability (spacefor-time substitution). This procedure often assumes spatiotemporal symmetry (Skøien and Blöschl, 2006; Harman et al., 2011) and is performed in such fields as regional hydrology (Hosking and Wallis, 1997; Peel and Blöschl, 2011), ecology (Pickett, 1989), geomorphology (Abrahams 1972). This symmetry is grounded on the Taylor hypothesis (Taylor, 1938), relating the time variation of a flow at a fixed point in a streamline with the spatial codependence between the flow changes at two fixed points along the same stream. However, the assumption of the Taylor Hypothesis is only legitimate along the same stream. The treatment of multiple streams is therefore not straightforward, which poses additional challenge to geostatistical time series analysis e.g. in regional hydrology.

Statistics over different time scales or over spatial and temporal dimensions would only yield similar results if the distribution were stationary and the system in equilibrium, e.g. lying in dynamical stable states. That would also imply that long term temporal statistics would correspond to wide spatial statistics (ergodicity and spatiotemporal symmetry). However, the hydrological system is not necessarily in equilibrium. Therefore, the relative rates of evolution at slow and fast time scales, the scale interaction feedback, needs to be taken into account when relating spatial and temporal features of the hydrological system.

Perdigão and Blöschl (2014) have tackled these problems by introducing statistical and dynamical methods that effectively quantify not only the role of individual flood drivers but also on their nonlinear interactions, including feedback mechanisms that ultimately result in emerging behaviour on floods not explainable by any of their individual drivers or their simple combinations. In doing so, the non-equilibrium dynamics have been brought out as coevolving processes in the hydrological system. Their role in the flood response to its interacting drivers has been analysed and modelled for a better understanding of the underlying mechanisms and interactions at play. Finally, a rate of coevolution has been introduced that enables space-for-time substitution in spatially extended systems with multiple streams, thus legitimising that procedure across multiple streams.

The present study builds on these advances from Perdigão and Blöschl (2014) and goes beyond them by evaluating the dynamics of the flood response to slow-fast landscapeclimate feedbacks and addressing spatiotemporal flood changes explicitly. For that purpose, a spatiotemporal sensitivity analysis is conducted in Sect. 2 , followed by the introduction and implementation of a dynamical model of slowfast landscape-climate coevolution (Sect. 3) and a dynamical model of the flood response to landscape-climate feedbacks (Sect. 4). The modelling results are also discussed in the respective sections (Sects. 3, 4) in connection to the diagnostic analysis of Sect. 2, closing the loop a statistical-dynamical manner. Concluding remarks are then laid out in Sect. 5.

\section{Spatiotemporal sensitivity analysis: (a)symmetry and coevolution}

Our analysis begins with a diagnostic evaluation of the flood response to the dynamically interacting landscape-climate system. For that purpose, a quantitative spatiotemporal sensitivity analysis is conducted whereby the spatial dimension represents the legacy of the slow, millennial time scale, and the temporal dimension refers to the fast, decadal time scale of the dynamics. In practice, the axes are built by sorting mean annual precipitation and mean regional precipitation in ascending order. Mean annual precipitation refers to the temporally averaged precipitation over a multidecadal time window, for each catchment. It thus provides a temporally averaged spatial distribution (space axis). Mean regional precipitation, in turn, refers to the regionally averaged precipitation for each year, thus providing a spatially averaged temporal distribution (time axis).

The data used come from a diverse pool of 804 catchments from Austria, spanning five hydroclimatic regions across the country, from Alpine to low-land catchments. This spatial diversity represents the legacy of the long-term millennial dynamics at which landform processes have coevolved with the hydroclimate system. The fast dynamics are extracted from a temporal window spanning from 1976 till 2008, with care to consider all partitions of that domain in order to avoid window-specific results for that period. That is, instead of considering just one window, a diversity of windows is considered within that domain in order to provide a more robust diagnosis. The same procedure is applied to the spatial analysis, by analysing multiple partitions of the set of catchments, in order to enrich the robustness of its diagnostic as well.

The spatial variability of precipitation is intimately related to the different catchment properties, such as elevation, with which it dynamically interacts. Therefore, the flood response to precipitation implicitly depends on the feedbacks between climatic and landscape processes, and is thus an indicator of their dynamical interaction, their coevolution.

Figure 1 depicts the spatiotemporal flood response to precipitation and underlying landscape-climate interactions, over the overall hydrological diversity across Austria. The presence of such interactions is diagnosed from the symme- 


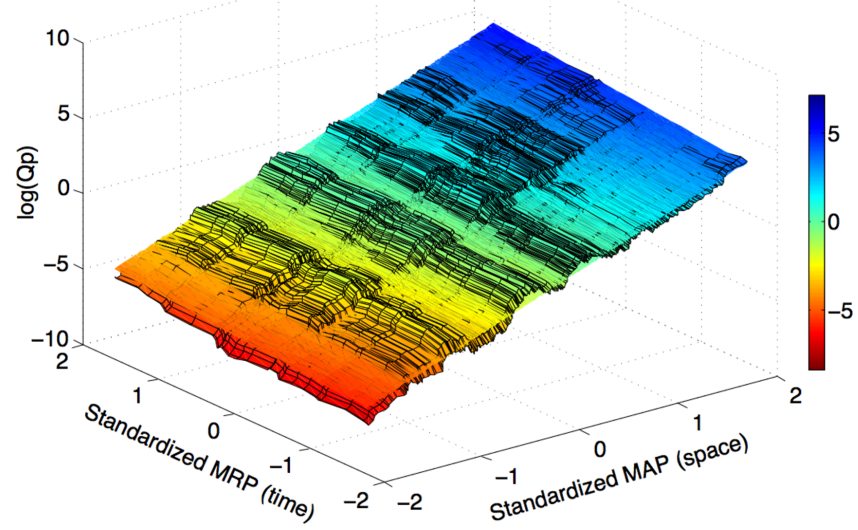

Figure 1. Spatiotemporal sensitivity of flood peaks to precipitation for Austria as a whole, depicted as surface plots of flood peaks $Q^{\mathrm{p}}$ with respect to precipitation sorted along space, mean annual precipitation (MAP), and time, mean regional precipitation (MRP). Also shown is the best gradient fitting plane, the slopes of which are given by the median of all gradients along space and time. The axes are standardized and given in base-10 logarithmic scale. From Perdigão and Blöschl (2014).

try properties between the spatial and temporal flood sensitivities to precipitation, as expressed by their spatial and temporal gradients. As noted in the introduction, a stable and stationary system would feature spatiotemporal symmetry, i.e. spatial and temporal gradients would match. However, as seen in Fig. 1 that is not the case. In fact, the spatial sensitivity of floods to precipitation is higher than the temporal sensitivity. Over Austria, a $10 \%$ spatial increase in precipitation leads to a $23 \%$ increase in flood peaks, whereas a $10 \%$ temporal increase in precipitation results in just $6 \%$ increase in flood peaks.

This symmetry break is an emerging property stemming from nonlinear dynamical feedbacks between landscape and climate, as noted in Perdigão and Blöschl (2014). A coevolution measure can then be introduced relating the spatiotemporal asymmetry with the underlying dynamics as expressed by relative characteristic celerities:

$\varepsilon=\alpha-\beta=f\left(\frac{\mathrm{d} s}{\mathrm{~d} t}\right)$,

where $\alpha$ and $\beta$ are respectively the medians of all spatial and temporal flood sensitivities to precipitation as calculated over all the possible partitions of the full spatial and temporal sets, and $f$ is a nonlinear function of the relative celerities $\mathrm{d} s / \mathrm{d} t$. The explicit dynamical form and derivation is given in Perdigão and Blöschl (2014).

Following the aforementioned methodological developments by Pires and Perdigão (2015), the coevolution can also be expressed as the nonlinear synergy (here Sn) between the spatial and temporal dimensions represented by the mean annual and mean regional precipitation axes (MAP,
MRP), leading to the same functional dependence on $\mathrm{d} s / \mathrm{d} t$ as in Eq. (1). The coevolution measure can thus be expressed equivalently as:

$\varepsilon=\operatorname{Sn}(\mathrm{MAP}, \mathrm{MRP})=f\left(\frac{\mathrm{d} s}{\mathrm{~d} t}\right)$.

This means that landscape-climate coevolution can now be diagnosed from nonlinear spatiotemporal interactions within precipitation itself. It is ultimately within these emerging nonlinearities that the landscape effects are implicitly reflected.

The rate of coevolution $\varepsilon$ is $1.70 \pm 0.38$ for all Austria (quantifying the asymmetry in Fig. 1). These rates have also been computed for the five Austrian hydroclimatic regions in particular as defined by Merz and Blöschl (2009), leading to rates close to 0 in the stable wet highlands of the Central Alps and on the stable dry lowlands of Eastern Austria, whilst around 1 in intermediate, more dynamically active regions such as the Southern Alps, Northern Alps and Northern Lowlands. These results further stress the notion that stable hydroclimatic regions are not coevolving significantly, whereas intermediate regions that have not yet settled in a stable state are dynamically evolving, thus producing the observed symmetry breaks.

\section{Nonlinear dynamics of landscape-climate coevolution}

The dynamics of landscape-climate coevolution are conceptualised as a stylised nonlinear dynamical model involving the evolving distributions of precipitation $(P)$ as representative of climate processes to which it is closely related and mean catchment elevation $(H)$ representing the dynamical legacy of landform processes.

The model is built taking the following key elements into consideration: (1) scale interactions (slow-fast landscapeclimate $(H, P)$ feedbacks); (2) limited resources: carrying capacity limiting $P$ to a maximum precipitable water limit $P_{\mathrm{C}}$; (3) stable states at lowlands and highlands and unstable coevolving hillslopes.

These considerations suggest a dynamical model of logistic type, where the fast dynamics are given by:

$\frac{\mathrm{d} P}{\mathrm{~d} t}=\gamma P\left(1-\frac{P}{P_{\mathrm{C}}}\right)$.

And the slow by:

$P_{\mathrm{C}}= \begin{cases}1-\exp \left(-\varepsilon \frac{H}{H_{l}-H}\right), & H<H_{l} \\ \exp \left[\varepsilon \frac{H_{l}-H}{H_{l}}\right], & H \geq H_{l}\end{cases}$

with $\varepsilon$ as in Eqs. (1) or (2). The term $\gamma$ corresponds to the transient evolution rate through internal entropy production in the dynamical system and $H_{l}$ is the elevation beyond which precipitable water becomes increasingly scarce. 


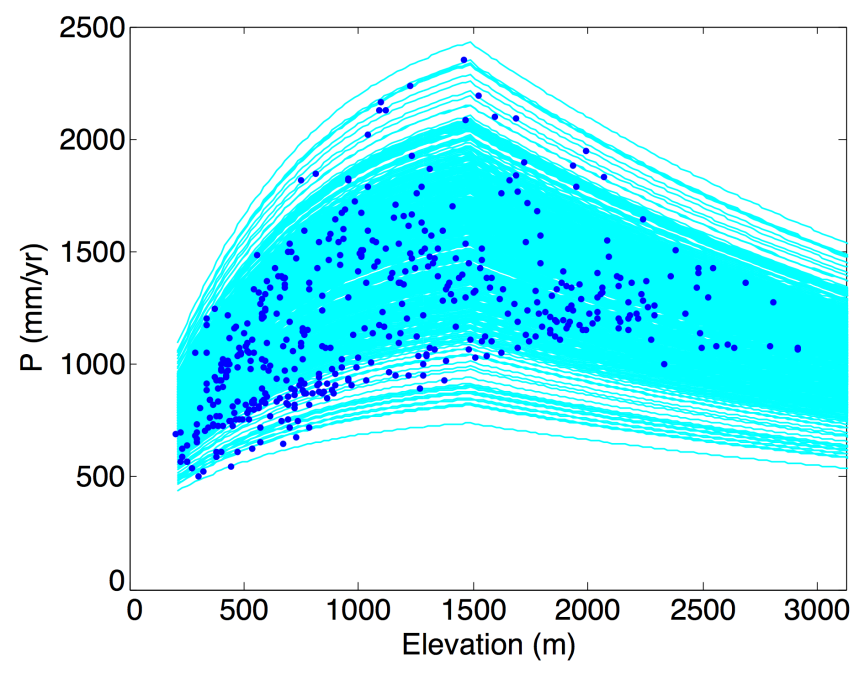

Figure 2. Mean annual precipitation $(P)$ with regards to mean catchment elevation. Blue dots represent $P$ for each catchment, as retrieved from the observational datasets (i.e. $P=$ MAP), and the solid lines represent the state space portrait of the dynamical model (Eqs. 3 and 4), considering a distribution of $P$ for each elevation. Reproduction of results from Perdigão and Blöschl (2014), albeit now with direct dynamical integration of a distribution instead of taking an ensemble of deterministic runs.

Whilst apparently simple, the model is able to capture complex dynamical behaviour. It does so by accounting for dynamical feedbacks between landscape and climate processes as represented by the intrinsic nonlinear interactions between $P$ and $H$. The key to the model ability to represent complexity thus lies in the way it conceptualises the interactions, i.e. on the model structure. It is also within its nonlinear structure that third-party processes are implicitly taken into account: in fact, their dynamical footprint, whilst not explicitly quantified as state variables, lies implicit within the nonlinear dynamics connecting $P$ with $H$.

In practical terms, the model dynamically prescribes a distribution for precipitation for a given elevation or at a given time. In other words, it provides a deterministic law for the evolution of a distribution, i.e. it is a stochastic-dynamic model. As such, it enables a dynamical understanding of nonstationary physical processes responsible for changes in precipitation regimes over space or time.

The model is validated against observational data in Fig. 2. There, the observed Precipitation $(P=\mathrm{MAP})$ for each mean catchment elevation $(H)$ is depicted as blue dots, while the model output prescribing the distribution of $P$ for each $H$ is portrayed as cyan lines.

While in Perdigão and Blöschl (2014) an ensemble of multiple realisations is taken in the model experiments, here the integration is performed directly in stochastic-physical mode, i.e. the evolution of the entire distribution is dynamically prescribed as a whole at once.
The results show a good agreement between model and observations, with the model-generated distribution $(H, P)$ aptly encompassing the data distribution $(H, P=\mathrm{MAP})$. These are fully consistent with those from Perdigão and Blöschl (2014). Moreover, now their comparison is further supported by the nonlinear codependence between model and data results as computed using Information Correlation (introduced by Pires and Perdigão in 2007) and Entropy Anamorphosis estimators (Pires and Perdigão, 2012, 2013), yielding an overall $84 \%$ agreement.

\section{Dynamical flood response to landscape-climate feedbacks}

The present section addresses the dynamical response of flood peaks to landscape-climate feedbacks. For that purpose, a stylised nonlinear dynamical model is built relating floods with the coevolving landscape-climate system. By taking the derivations from Perdigão and Blöschl (2014) into account, a dynamical equation for $Q^{\mathrm{p}}$ is obtained:

$\frac{\mathrm{d} Q^{\mathrm{p}}}{\mathrm{d} t}=\gamma \kappa Q^{\mathrm{p}}\left[1-B\left(Q^{\mathrm{p}}\right)^{1 / \kappa}\right]$

where

$B=\frac{P_{0}}{P_{\mathrm{C}}\left(Q_{0}^{\mathrm{p}}\right)^{1 / \kappa}}$,

where the product $\gamma \kappa$ is the transient evolution rate through internal entropy production of this system, with $\gamma$ as in the Precipitation system (Eq. 3).

Given a flood distribution at a given time or elevation, its distribution at another can be determined using the dynamical model. This has applications for spatiotemporal flood estimation. Rather than individual floods, the focus is given to distributions. While the intra-distribution dynamics can still be regarded as stochastic variables for simplicity, the "climatologies" can be handled deterministically, either as an ensemble of deterministic pointwise runs, or as deterministic prescriptions of the evolution of a flood distribution.

Figure 3 depicts the mean annual flood peaks $\left(Q^{\mathrm{p}}\right)$ in logarithmic scale with regards to mean catchment elevation. Blue dots represent $Q^{\mathrm{p}}$ for each catchment, as retrieved from the observational datasets, and the solid lines represent the state space portrait of the dynamical model (Eq. 5), considering a distribution of $Q^{\mathrm{p}}$ for each elevation, and coevolution rates $\varepsilon=1.70 \pm 0.38$. The integration procedure is akin to that performed for precipitation above.

The comparison between observations and model results suggests a reasonable agreement between them. In fact, the model output encompasses the observed states and their relative density in state space, with higher observational density being accompanied by higher model output density. These results are also consistent with those from Perdigão and Blöschl (2014) and now further supported by the nonlinear 


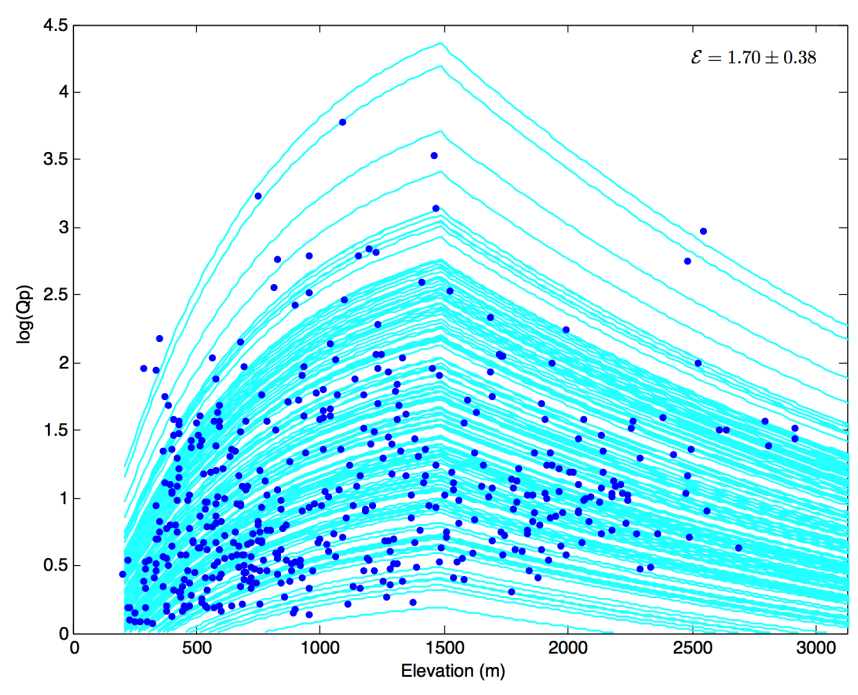

Figure 3. Mean annual flood peaks $\left(Q^{\mathrm{p}}\right)$ in logarithmic scale with regards to mean catchment elevation. Blue dots represent $Q^{\mathrm{p}}$ for each catchment, as retrieved from the observational datasets, and the solid lines represent the state space portrait of the dynamical model (Eqs. 5 and 6), considering a distribution of $Q^{\mathrm{p}}$ for each elevation, and coevolution rates $\varepsilon=1.70 \pm 0.38$. Reproduction of results from from Perdigão and Blöschl (2014), albeit now with direct dynamical integration of a distribution instead of taking an ensemble of deterministic runs.

measure of Information Correlation between model output and observations, yielding an overall $71 \%$ agreement.

At first sight, the spread of flood peaks for each given elevation might cause concern. However, as noted before, the model prescribes the dynamical evolution of a distribution, not of an individual flood event. As such, for each elevation there will be not one flood peak, but rather a distribution of flood peaks. This distribution will naturally be different for different elevations, depending on the coevolving landscapeclimate dynamics.

The spread of the flood distribution, seen in both model and observations, is higher in coevolving intermediate elevations, which is consistent with the associated unstable dynamical behaviour and consequent entropy production. The converse holds for stable lowlands and highlands, where the observed and modelled lower spread is consistent with more stable dynamical regimes associated with weak landscapeclimate coevolution.

\section{Conclusions}

Floods have been shown to respond to landscape-climate feedbacks in a nonlinear dynamical manner, whereby the flood distribution nonlinearly interacts with the coevolving landscape-climate system over space and time. The coevolving dynamics have been associated with emerging spatiotemporal symmetry breaks in the flood response to precipita- tion. In fact, in coevolving regions flood peaks change in a more responsive manner to changes in precipitation over space than over time. For instance, over Austria a $10 \%$ spatial increase in precipitation leads to a $23 \%$ increase in flood peaks, whereas a $10 \%$ temporal increase in precipitation results in just $6 \%$ increase in flood peaks.

The eventual role of year-to-year carryover and consequent memory accumulation has been ruled out, whereas the coevolution hypothesis gained strength with nonlinear statistical and dynamical analysis and modelling relating flood evolution with nonlinearly interacting landscape-climate dynamics.

A coevolution rate index has been introduced relating the emerging spatiotemporal asymmetry with relative celerities: stable non-coevolving areas exhibit symmetry between spatial and temporal sensitivitities of floods to precipitation, whereas coevolving hillslopes exhibit symmetry breaks in those sensitivities, triggered by the intrinsic nonlinear dynamical feedbacks between landscape and climate processes.

This study has further stressed the need to take into account, in space-for-time trading in regional hydrology, the nonlinear feedbacks between dynamically interacting (coevolving) processes through relative celerities. Rather than an obstacle, nonlinearities bring in extra information that ultimately legitimates such techniques and enables a better understanding of the overall dynamics of the flood response to the landscape-climate system.

Last but not least, the study has brought to light nonlinear dynamical interactions among floods and drivers, resulting in emerging effects not attributable to any of the individual factors involved but rather to the overall nonlinear dynamical feedbacks: changes in the flood peak distribution (nonstationarity) and symmetry breaks in its spatiotemporal response to precipitation (spatiotemporal asymmetry). In effect, a slow-fast nonlinear dynamical flood change model has been presented and evaluated, relating the dynamical evolution in the flood distribution with nonlinear interactions among intervening processes.

Acknowledgements. This research was supported by the ERC Advanced Grant "Flood Change", project 291152.

\section{References}

Abrahams, A. D.: Environmental constraints on the substitution of space for time in the study of natural channel networks, Geol. Soc. Am. Bull., 83, 1523-1530, 1972.

Hall, J., Arheimer, B., Borga, M., Brázdil, R., Claps, P., Kiss, A., Kjeldsen, T. R., Kriaučiūnienè, J., Kundzewicz, Z. W., Lang, M., Llasat, M. C., Macdonald, N., McIntyre, N., Mediero, L., Merz, B., Merz, R., Molnar, P., Montanari, A., Neuhold, C., Parajka, J., Perdigão, R. A. P., Plavcová, L., Rogger, M., Salinas, J. L., Sauquet, E., Schär, C., Szolgay, J., Viglione, A., and Blöschl, G.: Understanding flood regime changes in Europe: a 
state-of-the-art assessment, Hydrol. Earth Syst. Sci., 18, 27352772, doi:10.5194/hess-18-2735-2014, 2014.

Harman, C. J., Troch, P. A., and Sivapalan, M.: Functional model of water balance variability at the catchment scale: 2 . Elasticity of fast and slow runoff components to precipitation change in the continental United States, Water Resour. Res., 47, W02523, doi:10.1029/2010WR009656, 2011.

Hosking, J. R. M. and Wallis, J. R.: Regional Frequency Analysis: An approach Based on L-Moments, 224 pp., Cambridge Univ. Press, Cambridge, UK, 1997.

Merz, R. and Blöschl, G.: A regional analysis of event runoff coefficients with respect to climate and catchment characteristics in Austria, Water Resour. Res., 45, W01405, doi:10.1029/2008WR007163, 2009.

Peel, M. C. and Blöschl, G.: Hydrologic modelling in a changing world, Prog. Phys. Geogr., 35, 249-261, 2011.

Perdigão, R. A. P. and Blöschl, G.: Spatiotemporal flood sensitivity to annual precipitation: Evidence for landscapeclimate coevolution, Water Resour. Res., 50, 5492-5509, doi:10.1002/2014WR015365, 2014.

Pickett, S. T. A.: Space-for-time substitution as an alternative to long-term studies, in Long-Term Studies in Ecology: Approaches and Alternatives, edited by: Likens, G. E., 110-135, Springer, NY, 1989.
Pires, C. A. and Perdigão, R. A. P.: Non-Gaussianity and asymmetry of the winter monthly precipitation estimation from the NAO, Mon. Weather Rev., 135, 430-448, doi:10.1175/MWR3407.1, 2007.

Pires, C. A. L. and Perdigão, R. A. P., Minimum mutual information and non-Gaussianity through the maximum entropy method: Theory and properties, Entropy, 14, 1103-1126, doi:10.3390/e14061103, 2012.

Pires, C. A. L. and Perdigão, R. A. P.: Minimum mutual information and non-Gaussianity through the maximum entropy method: Estimation from finite samples, Entropy, 15, 721-752, doi:10.3390/e15030721, 2013.

Pires, C. A. L. and Perdigão, R. A. P.: Non-Gaussian interaction information: estimation, optimization and diagnostic application of triadic wave resonance, Nonlin. Processes Geophys., 22, 87108, doi:10.5194/npg-22-87-2015, 2015.

Skøien, J. and Blöschl, G.: Catchments as space-time filters-A joint spatiotemporal geostatistical analysis of runoff and precipitation, Hydrol. Earth Syst. Sci., 10, 645-662, doi:10.5194/hess10-645-2006, 2006.

Taylor, G. I.: The Spectrum of Turbulence, Proc. R. Soc. Ldn., Ser. A, 164, 476-490, doi:10.1098/rspa.1938.0032, 1938. 